\title{
A NUMERICAL STUDY ON THE ENDURANCE LIMIT OF PARTICULATE REINFORCED METAL MATRIX COMPOSITES (PRMMCS) USING THE DIRECT METHOD AND THE STATISTICAL LEARNING
}

\author{
Geng Chen $^{1}$, Bezold Alexander ${ }^{1}$, Christoph Broeckmann ${ }^{1}$, and Dieter Weichert ${ }^{2}$ \\ ${ }^{1}$ Institute for Materials Applications in Mechanical Engineering, RWTH Aachen University \\ Augustinerbach 4, Aachen, Germany \\ e-mail: \{g.chen, a.bezold, c.broeckmann\}@iwm.rwth-aachen.de \\ ${ }^{2}$ Institute of General Mechanics, RWTH Aachen University \\ Templergraben 64, Aachen, Germany \\ e-mail: weichert@iam.rwth-aachen.de
}

Keywords: Direct Method, RVE, Metal Matrix Composite (MMC), Statistics, Classification

\begin{abstract}
Particulate reinforced metal matrix composites (PRMMCs) are characterized by their stochastic and irregular microstructure. The strength of the material has been noticed to have a large scatter and great dependence on the underlying composite structure. In order to understand how size and distribution of the reinforcement particles contribute to the effective material behaviors, we elaborated in present study a numerical approach which incorporates homogenization technique, shakedown analysis, and statistical learning. To demonstrate this approach, a typical PRMMC material, WC - $20 \mathrm{wt} . \%$ Co, was taken as example and numerous representative volume element (RVE) samples of this material were built based on both real and artificial microscope images. Multiple effective behaviors, including the ultimate strength and endurance limit, were evaluated on each RVE sample using the direct method. In order to understand how multiple structural factors jointly affect the overall composite strength, the predicted strength and other selected features extracted from the samples are submitted to few statistical models such as logistic regression and artificial neural network (ANN) to establish predictive models. On the basis of these models we discussed how few structural factors, which have been identified to have nontrivial impact, contribute to the endurance limit of the selected PRMMC material.
\end{abstract}




\section{INTRODUCTION}

Particulate reinforced metal matrix composites (PRMMCs) are becoming increasingly popular for being used as structural materials due to their preferable mechanical properties such as high strength and good wear resistance. Compared to fiber reinforced composites, mechanical behaviors of PRMMCs are more isotropic, and the materials are in general less expensive to be fabricated. As composed of metal matrix and discrete ceramic particles, the microstructure of the PRMMCs are irregular and highly randomized. Therefore, the mechanical behaviors of the material, including the strength, have been noticed to have a large scatter and great dependence on the underlying composite structure. In order to enhance the economic use of the material and accelerate the development period of new PRMMCs compositions, it is essential to have an engineering tool for predicting the strength of materials from their microstructural morphologies and identifying the reasons contributing to the material strength; and this is the objective of the present paper.

One common practice for determining the macroscopic behaviors of composite materials is through numerical homogenization. According to this approach, finite element (FE) models of the composite structure, the so called representative volume element (RVE), should be built; then by applying loads on it and deriving the effective terms of its responses, the global behaviors of the composite can be obtained. Beyond determining the mechanical behaviors such as elasticity, numerical homogenization has been used also for determining the strength of the composite materials. To do this, one viable way is to apply the direct method (DM) to RVE models and converting the obtained results to the macroscopic strengths. Previously, Weichert et al. [1] have elucidated in their studies the mathematical formulation of this approach based on the static theorem, while Chen $\mathrm{H}$. and Pontor [2] proposed a formulation serving the same aim but based on the kinematic theorem. By applying these methods to composites reinforced by periodically aligned fibers, the in-plane strength of the material have been successfully calculated. The availability of this technique has been extended in our previous studies to random heterogeneous materials [3]. For random heterogeneous materials such as PRMMCs, one intrinsic difficulty is to represent the material by RVE models. To have a model which smears out the influence raised by the random size and the distribution of the reinforcement particles, it requires the size of the model to be exceptionally large and this will cause sever computational difficulty. One feasible remedy to this problem is, instead of using one RVE, employing many RVEs which are considered to be statistically equivalent. In the light of this idea, an in-house code was developed for simplify the modeling of RVE samples by automatically converting SEM images to adaptively meshed RVE models. Taking advantage of this code, we performed DM on many samples converted from SEM image of a typical PRMMC material, WC - $20 \mathrm{wt} . \%$ Co, and obtained the statistics of their macroscopic ultimate strengths and endurance limits.

As has been addressed, the interests of studying the strength of PRMMCs is not only confined to determining the strength; indeed, it is also important to have an insight of factors contributing to the strength. To expose the relationship between the composite structure and the material strength, one advisable solution is through statistical learning. Statistical learning is a fast evolving discipline in recent years. Many algorithms arisen from this field have been successfully implemented for solving challenging medical and engineering problems. The problems of statistical learning can be coarsely categorized to supervised and unsupervised. In supervised learning, the goal is to predict the value of an outcome measure based on a number of input measures; in unsupervised learning, there is no outcome measure, and the goal is to describe the associations among a set of input measures. Depending on if the outputs are qualitative or 
quantitative, problems of the supervised learning can be further subdivided to regression and classification (or pattern recognition). Although problems belonging to unsupervised learning prevail in engineering practices, most of the studies dedicated to applying statistical learning to engineering problems focus on the supervised learning. Some achievements made in this respect can be seen from monograph [4] and review articles [5, 6, 7]. In the scope of composite structures and their mechanical behaviors, recently the studies where statistical learning is employed as the main approach increase in both number and the diversity [8,9]. Among these studies, the application of learning models to either experimental or simulated data gradually becomes a new paradigm for results interpretation and knowledge discovery.

In the present study, beside determining the strength of a PRMMC material, WC - $20 \mathrm{wt} . \%$ $\mathrm{Co}$, one additional objective is to understand how multiple structural factors jointly affect the overall composite endurance limit. To answer this question, we constructed predictive models based on both logistic regression and back propagation artificial neural network (ANN). Based on analyzing results of direct method using these models, the relationship between endurance limit and selected micromechanical measurements will be discussed.

\section{SHAKEDOWN OF RVE MODELS}

To study the effective behaviors of heterogeneous materials, the material is reflected in two scales: the microscopic scale is small enough for the heterogeneities to be identified. The macroscopic scale is large enough for the heterogeneities to be expelled. The two scales are well-separated and they are described by two coordinate systems: the global coordinate system $\boldsymbol{x}$ and local coordinate system $\boldsymbol{y}$. The following relationship holds

$$
\boldsymbol{y}=\frac{\boldsymbol{x}}{\epsilon} \text {. }
$$

$\epsilon$ is a small scale parameter which determines the size of the RVE.

For a heterogeneous meterial, once submitted to an external loading, its microscopic stress field $\boldsymbol{\sigma}$ in $\boldsymbol{y}$ and its macroscopic counterpart $\boldsymbol{\Sigma}$ satisfy the relationship

$$
\boldsymbol{\Sigma}=\frac{1}{\Omega} \int_{\Omega} \boldsymbol{\sigma}(y) d V=\langle\boldsymbol{\sigma}(\boldsymbol{y})\rangle .
$$

Here $\langle\cdot\rangle$ stands for the averaging operator, and $\Omega$ indicates the RVE domain. Similarly, the relationship between strain measures satisfies

$$
\boldsymbol{E}=\frac{1}{\Omega} \int_{\Omega} \varepsilon(y) d V=\langle\varepsilon(\boldsymbol{y})\rangle .
$$

The local strain $\varepsilon$ can be decomposed into two parts: The average value $\boldsymbol{E}$ and a fluctuating part $\varepsilon^{*}$

$$
\varepsilon(\boldsymbol{u})=\boldsymbol{E}+\varepsilon^{*}
$$

When all constituents of a RVE are elastic, the overall behavior of the RVE is elastic as well. In this circumstance, $\boldsymbol{\Sigma}$ and $\boldsymbol{E}$ are correlated by an effective elastic tensor $\overline{\mathbb{C}}$

$$
\boldsymbol{\Sigma}=\overline{\mathbb{C}}: \boldsymbol{E} .
$$

In case that the heterogeneous material to be considered behaves isotropically in the macro scale, same to the single phase material, $\overline{\mathbb{C}}$ can be uniquely determined from two elastic constants, such as effective Young's modulus $\bar{E}$ and effective Poission's ratio $\bar{\nu}$. 
When the composite material is composed of elasto-plastic constituents, its macroscopic ultimate strength $\Sigma_{U}$ and endurance limit $\Sigma_{\infty}$, which correspond to plastic and shakedown limit in the micro scale, can be studied by incorporating homogenization with direct method. As formulated by Magoariec et al. [10], when the shakedown state is attained in the micro scale, stress field pertained to the reference elastic body $\mathcal{B}^{E}, \sigma^{e}$, and the time invariant residual stress field $\bar{\rho}$ are required to satisfy following conditions

$$
\begin{gathered}
\boldsymbol{\sigma}^{e}: \begin{cases}\boldsymbol{\nabla} \cdot \boldsymbol{\sigma}^{e}=0 & \text { in } \Omega, \\
\boldsymbol{\sigma}^{e}=\mathbb{C}:\left(\boldsymbol{E}+\boldsymbol{\varepsilon}^{*}\right) & \text { in } \Omega, \\
\boldsymbol{\sigma}^{e} \cdot \boldsymbol{n} & \text { anti-periodic on } \partial \Omega, \\
\boldsymbol{u}^{*} & \text { periodic on } \partial \Omega, \\
\langle\varepsilon\rangle=\boldsymbol{E} . & \end{cases} \\
\overline{\boldsymbol{\rho}}: \begin{cases}\boldsymbol{\nabla} \cdot \overline{\boldsymbol{\rho}}=0 & \text { in } \Omega, \\
\overline{\boldsymbol{\rho}} \cdot \boldsymbol{n} & \text { anti-periodic on } \partial \Omega .\end{cases}
\end{gathered}
$$

Here, $\Omega$ indicates the RVE domain, $\partial \Omega$ the surface, $\boldsymbol{n}$ the outer normal, and $\boldsymbol{u}^{*}$ the fluctuation part of the displacement corresponds to $\varepsilon^{*}$.

Although shakedown problem in the RVE scale can be studied by either strain or stress approach [10], in present study we consider exclusively the stress approach. For stress approach the load prescribed on RVE is the macroscopic stress $\Sigma$. Because the material to be studied is non-periodic, a small specification is made on conditions (6) and (7), where, instead of enforcing the node-wise anti-periodicity of the residual stresses and periodicity of the fluctuating displacement, we apply the statically uniform boundary conditions (SUBC) on the purely elastic reference RVE. As a consequence, the shakedown problem yields $\overline{\boldsymbol{\rho}} \cdot \boldsymbol{n}=\mathbf{0}$ on $\partial \Omega$ and one can easily prove that, in the absence of the body force $\langle\overline{\boldsymbol{\rho}}\rangle=\mathbf{0}$, so $\overline{\boldsymbol{\rho}}$ does not contribute to the macroscopic stress.

By discretizing the physical fields in (6) and (7) by means of the FE formulations, the application of the static theorem to RVEs composed of elastic perfectly plastic materials leads to following optimization problem:

$$
\begin{array}{ll}
\underset{\overline{\boldsymbol{\rho}}, \alpha}{\operatorname{minimize}} & -\alpha \\
\text { subject to } & \sum_{i=1}^{N G} \boldsymbol{C}_{i} \overline{\boldsymbol{\rho}}_{i}=\mathbf{0}, \\
& F\left(\alpha \boldsymbol{\sigma}_{i k}^{e}+\overline{\boldsymbol{\rho}}_{i}\right)-\sigma_{Y i}^{2} \leq 0 \\
& \forall i \in[1, N G] ; k \in[1, N V] .
\end{array}
$$

Here, $\alpha$ is referred to as the load factor, $\boldsymbol{C}$ the equilibrium matrix, $\overline{\boldsymbol{\rho}}_{i}$ the stress tensor associated with the $i^{\text {th }}$ Gaussian point, $\boldsymbol{\sigma}_{i k}^{e}$ the abbreviation of $\boldsymbol{\sigma}_{i}^{e}\left(\boldsymbol{P}_{k}\right)$ which means the $\boldsymbol{\sigma}^{e}$ at Gaussian point $i$ and load vertex $k, \sigma_{Y}$ the yield strength, $F$ the yield function, $N G$ the number of Gaussian points, and $N V$ the number of vertices. Solving (8) yields the load capacity of the RVE, and depending on if $k=1$ or $k>1$ the calculated strength corresponds to either plastic limit or endurance limit. In the present study, the load scenario considered is restricted to non-reversed uniaxial stress, in this case $N V=2$ and $\boldsymbol{\sigma}_{i k}^{e}=0$ for all $k=2$. 


\section{CLASSIFICATION MODELS}

After solving (8) pertained to each RVE and obtaining $\Sigma_{\infty}=\alpha \cdot \Sigma$, the results are fed to statistical models for gaining a better interpretation. The statistical models considered in the present study are classification models. There are many advantages for having classification models: First, classification models are robust; our observations convinced that these models are insensitive to RVE size and sample size. Next, classification models have great practical values. Because material designers often face the problem of comparing between different composite structures, it is beneficial to have simple and inexpensive models for evaluating strength in an relative sense. However, it is worthy to note, that the purpose for introducing the predictive statistical model is not to replace the DM calculation. In fact, the study addresses the use of predictive model as a mean to understand the data; in other words, the interpretability of the model is accentuated compared to its predictivity.

We consider two classification models: the logistic regression and the ANN, both of them determine from a large set of observations $\left(X_{1}, Y_{1}\right),\left(X_{2}, Y_{2}\right), \ldots\left(X_{m}, Y_{m}\right)$ by which $Y_{m}$ are categorical variables, to which class $Y_{i}$ a new observation $X_{i}$ would belong to at its highest probability. Although both logistic regression and ANN serve the same goal, in comparison the former one is convenient to be explained but lacks sufficient flexibility in prediction, while the latter one renders excellent prediction but is hard to be interpreted. The algorithms of both models, as well as the strategies adopted to avoid overfitting, are far beyond the scope of the present paper. More detailed explanations on these issues can be found in [11].

\section{FEATURE SELECTION FOR THE STATISTICAL MODELS}

A key factor for having a well performed predictive model is that features $X$ are properly selected. In the present case, the simplest way to select $X$ is to use RVE images directly as features. Although this approach prevails in the computer science community, it is not adopted because such feature is physically less interpretable. On selecting features for the statistical model, the first set of features adopted are Vol.\% WC, $\bar{E}, \bar{\nu}$, as well as peak valued equivalent elastic stress in both phases $\sigma_{W C, \max }^{e}$ and $\sigma_{C o, \max }^{e}$. Additionally, as shown in Figure 1, we noticed from few samples that $\Sigma_{\infty}$ is related to the distribution of the stress and thus features reflecting the distribution characteristics of the stress field should be incorporated into the model. Because total stress $\boldsymbol{\sigma}=\alpha \boldsymbol{\sigma}^{e}+\overline{\boldsymbol{\rho}}$ is unknown before solving the shakedown problem, features were extracted from the elastic stresses field instead.

The features chosen for representing the distribution characteristics of the stress field is highly stressed volume fraction $\left(V_{e f f}\right)$ defined as

$$
V_{e f f}^{n \% \sigma}=\frac{\sum_{i=1}^{N G} V_{i} \cdot \mathcal{H}\left(\sigma_{i}, n \% \sigma_{\max }^{e}\right)}{\sum_{i=1}^{N G} V_{i}} .
$$

Here $V_{i}$ is the volume and $\sigma_{i}$ the equivalent stress at Gaussian point $i$; $\mathcal{H}$ the Heaviside function defined as

$$
\mathcal{H}\left(\sigma_{i}, n \% \sigma_{\text {max }}^{e}\right)= \begin{cases}1, & \sigma_{i} \geq n \% \sigma_{\max }^{e} \\ 0, & \text { Oherwise }\end{cases}
$$

One can see from (9), that $V_{e f f}^{n}$ sums over volumes whose stress exceeds the threshold value $n \% \sigma_{\max }^{e}$, therefore by adjusting the magnitude of $n$ the stressed volume at each level can be easily accessed. In the present study, $V_{e f f}^{n \% \sigma}$ was evaluated for each phase separately, where 


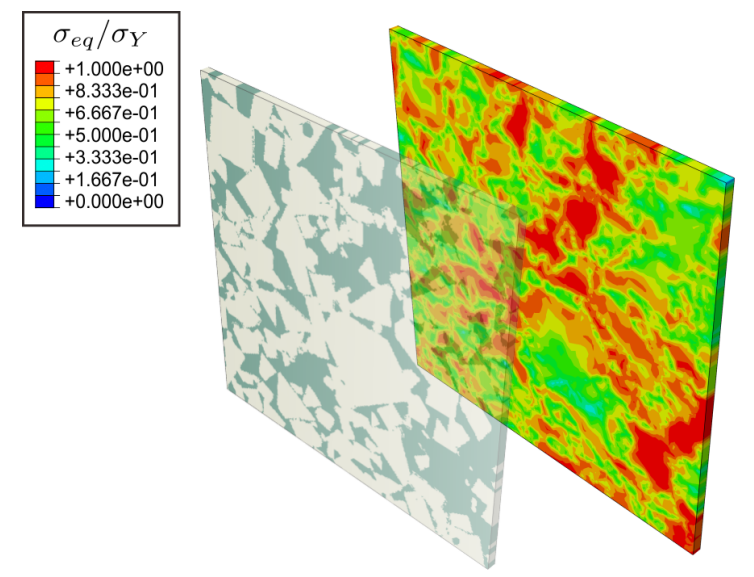

(a) $Z_{\Sigma_{\infty}}=1.50$

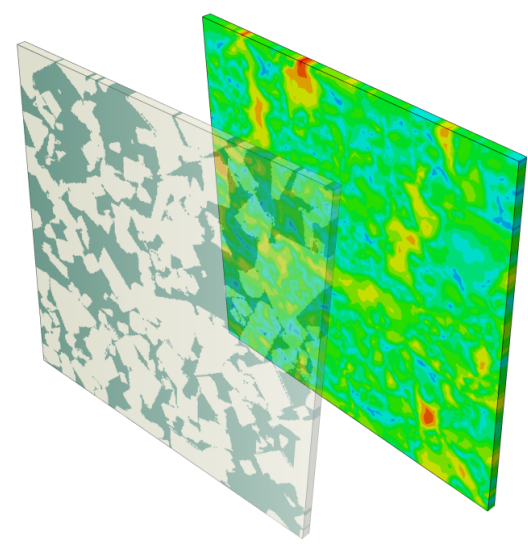

(b) $Z_{\Sigma_{\infty}}=-1.49$

Figure 1: Endurance limit and highly stressed volume

$\sigma_{\max }^{e}$ in (10) refers to $\sigma_{W C, \max }^{e}$ or $\sigma_{C o, \max }^{e}$, and calculation renders $V_{C o, e f f}^{n \% \sigma}$ or $V_{W C, \text { eff }}^{n \% \sigma}$. The calculation fixed $n$ to multiple values, where $n \in\{10,20, \ldots, 90\}$ and results were collected into a vector.

In summary, the features selected for the predictive models can be divided into two categories:

- Category 1 (Homogenized material parameter): WC Vol.\%, $\bar{E}, \bar{\nu}$

- Category 2 (Results of elastic simulation): $\sigma_{\max }^{e}, V_{e f f}^{n \% \sigma}$

\section{RESULTS AND DISCUSSIONS}

The numerical study is based on $50040 \mu m-40 \mu m$ - $1 \mu m$ RVE samples. Each sample is modeled from an individual artificial morphology generated by a simple random sequential adsorption (RSA) algorithm as shown in Figure 2. The algorithm is developed in Matlab [12] on the matrix basis. According to this algorithm, the RVE domain is initialized as a zero matrix and the program continuously projects prism shaped geometry into this matrix. After each projection, zero elements in the matrix are set to one if they belong to the prism domain and remain zero otherwise. The value of elements will not be reset if they have already been picked in previous iterations. Parameters controlling the projection, such as prism size, rotation angle, and center of the projection, are all random numbers. In order to be consistent with real WCCo microstructures, the algorithm adopts a configuration that $d_{W C}$ obeys a normal distribution with mean value $3 \mu \mathrm{m}$ and standard deviation $0.8 \mu \mathrm{m}$. The position where each particle locates is independent from the others and therefore there is no predefined clustering.

The models were built in commercial FE solver ABAQUS [13] and meshed by a uniform mesh configuration: the element type is fixed to linear wedge elements (C3D6); elements covering non-critical regions were assigned with a global size of $0.8 \mathrm{~m}$; while elements near the phase boundaries are of a finer density with an edge size of $0.2 \mathrm{~m}$. Under this configuration, the number of elements for each RVE sample varies between 15,000-20,000. In all models, both constituents are considered as elastic-perfect plastic materials with parameters given in Table 5 . Meanwhile, the study is restricted to small deformation and assumes plastic failure as the only failure mechanism.

RVEs are prescribed by a global uni-axial stress $\Sigma=10 \mathrm{MPa}$ for calculating $\boldsymbol{\sigma}^{e}$. After $\boldsymbol{\sigma}^{e}$ has been calculated, results were output to MATLAB together with geometrical set up to construct 


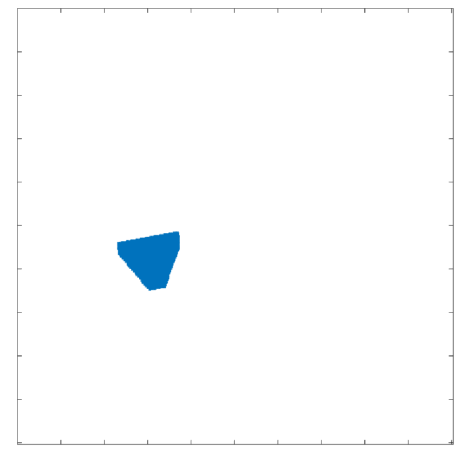

(a) Iteration $=1$

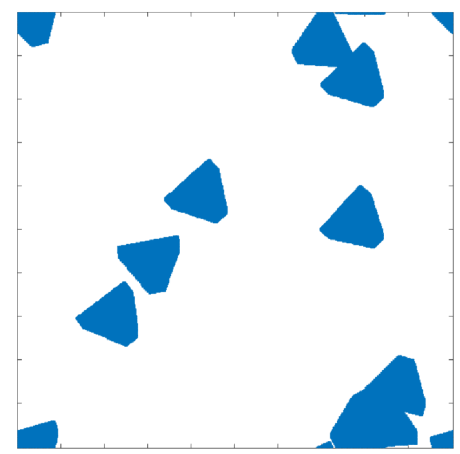

(c) Iteration $=10$

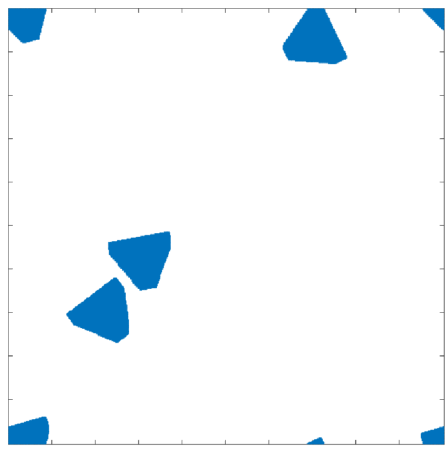

(b) Iteration $=4$

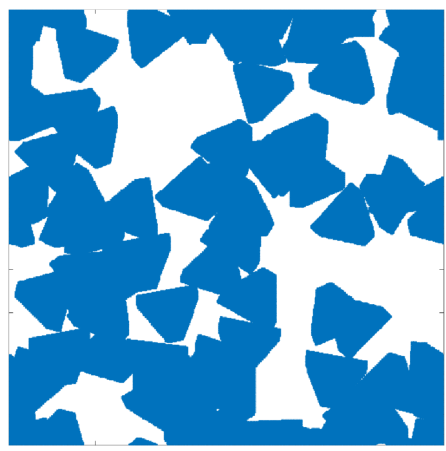

(d) Final

Figure 2: Inclusion process with fixed grain size

\begin{tabular}{cccc}
\hline & $E[\mathrm{GPa}]$ & $\nu[-]$ & $\sigma_{Y}[\mathrm{MPa}]$ \\
\hline $\mathrm{WC}$ & 700 & 0.24 & 2000 \\
$\mathrm{Co}$ & 210 & 0.30 & 683
\end{tabular}

Table 1: Material properties of both phases

optimization problem. Once these optimization problems were built and solved by an interior point method based solver Gurobi [14], the solutions were further used for building classification models. Two classification models used, namely the logistic regression and the ANN, were realized in the statistics and machine learning toolbox of the MATLAB. In the logistic regression model, input data are linear, squared, and interaction terms of aforementioned features. In the ANN classifier, model parameters are determined based on evidences gained from both parametric study and comparison of the cross validation errors between different model settings. In the end, a single hidden layer model consisted of 20 neurons in the hidden layer has been chosen as the ANN structure.

The criterion adopted in classification models are $\Sigma_{\infty}$ where the $Z$ score is defined as

$$
Z=\frac{x-\bar{x}}{s}
$$




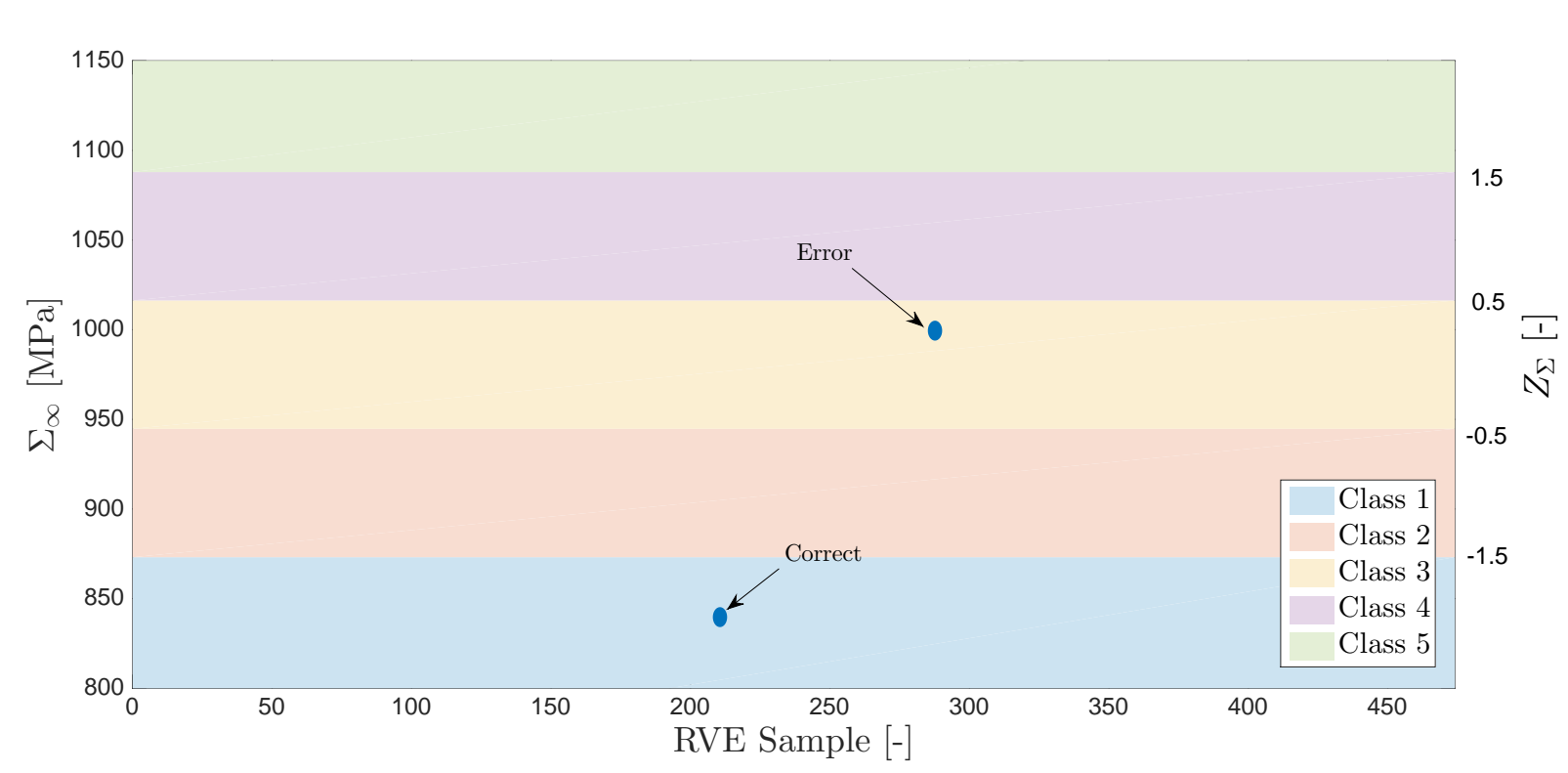

Figure 3: Domain of classes for the classification problems

Here $\bar{x}$ and $s$ indicate the first and second moment of the sample set, respectively. According to $Z$ score, the entire span of $\Sigma_{\infty}$ is sub-divided into 4 or 5 classes as follows

$$
\begin{gathered}
4 \text { Classes }\left\{\begin{array}{l}
\text { Class 1: } Z_{\Sigma_{\infty}}<-1.0 \\
\text { Class 2: }-1.0<Z_{\Sigma_{\infty}} \leq 0 \\
\text { Class 3: } 0<Z_{\Sigma_{\infty}} \leq 1.0 \\
\text { Class 4: } Z_{\Sigma_{\infty}}>1.0
\end{array}\right. \\
5 \text { Classes }\left\{\begin{array}{l}
\text { Class 1: } Z_{\Sigma_{\infty}}<-1.5 \\
\text { Class 2 : }-1.5<Z_{\Sigma_{\infty}} \leq-0.5 \\
\text { Class 3 : }-0.5<Z_{\Sigma_{\infty}} \leq 0.5 \\
\text { Class 4 : } 0.5<Z_{\Sigma_{\infty}} \leq 1.5 \\
\text { Class 5 : } Z_{\Sigma_{\infty}}>1.5
\end{array}\right.
\end{gathered}
$$

Herein, the five-classes problem, as schematically presented in Figure 3, is only considered upon the success of the four-classes problem.

In all predictive models the feature $\mathrm{x}$ is normalized as follows before submitted to a learning algorithm

$$
x_{i}^{\prime}=2 \cdot \frac{x_{i}-\min (x)}{\max (x)-\min (x)}-1 .
$$

This way, the normalized feature $x_{i}^{\prime} \in[-1,1]$, and the model will not face the numerical difficulty caused by the poor scaling.

To demonstrate the result of classification, we plot the sample point in the position where it actually locates with the color corresponding to the predicted class. When decision of a classification model is correct, then the color of a sample overlaps with its background color (see Figure 3 ). The wrong classification means the strength of a sample is either over- or underestimated. The overestimation of the strength is refereed to as false positive (FP), while underestimation as false negative (FN). Comparatively, FP is riskier than FN. According to the definition (12-13), the classes are aligned continuously and thus a certain amount of the classification tolerance should be allowed. In other words, if a sample lies closely enough to the classification boundary, then the wrong classification of the sample to the adjacent class should not be counted in 
the error metrics. To exclude this particular typed error from the error metrics, we set tolerance to $1 / 10 \cdot s$ and employed modified error rate as the measurement of the classification error.

In the course of building statistical models for classifying $\Sigma_{\infty}$, we noticed that the highly stressed volume fraction $V_{e f f}^{n \% \sigma}$ plays a critical role. When the features in Category 2 were omitted, the results of classification is similar to Figure 4 where the overall error rate is $40.5 \%$. Also, there is hardly any improvement in performance when model parameters were tuned or an alternative learning algorithm was used. The result in Figure 4 shows that the model without features in Category 2 fails to classify a sample into a correct strength class.

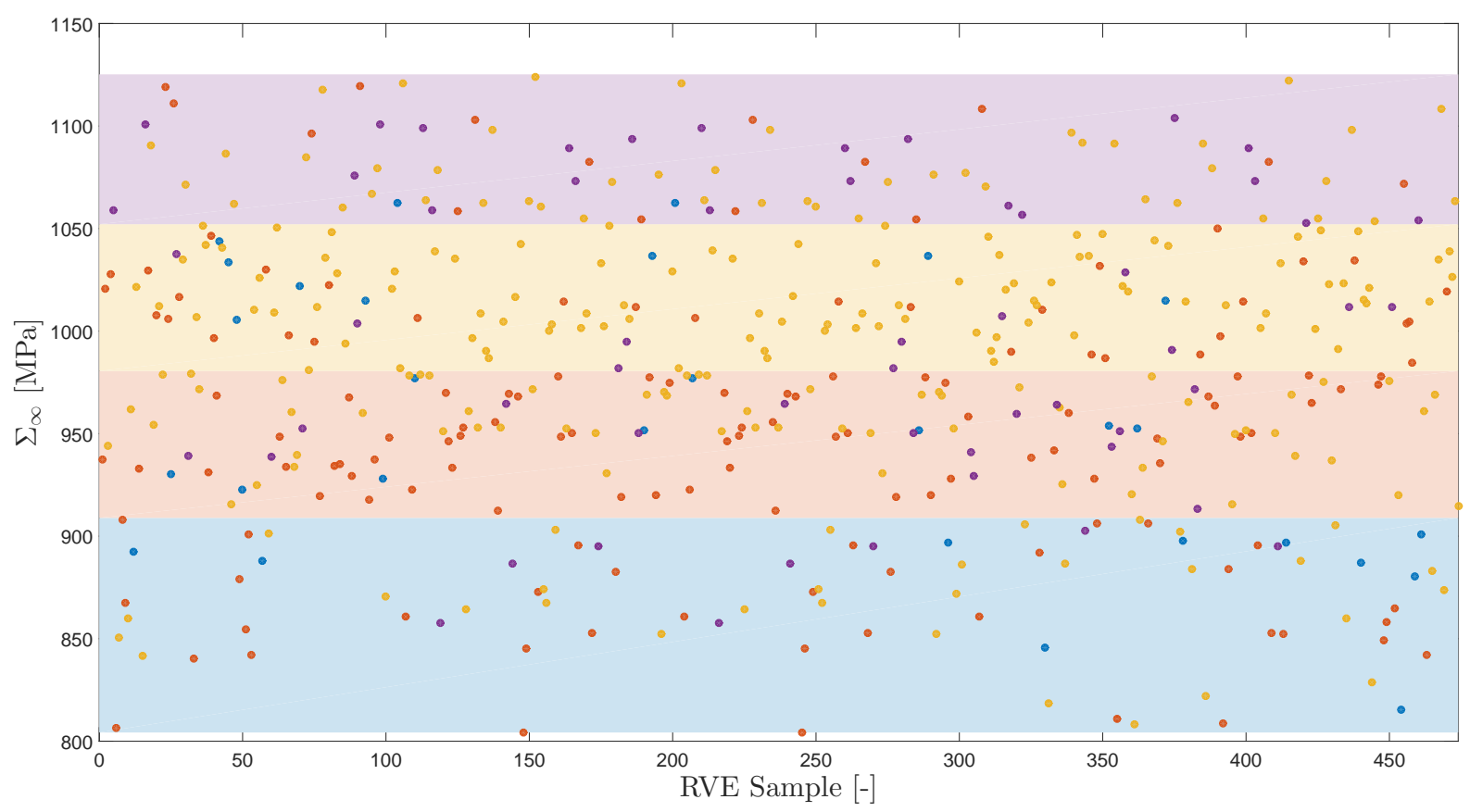

Figure 4: Results of an ANN classification model on $\Sigma_{\infty}$ without features in Category 2

After the features in Category 2 were introduced back to the model, we observed that even without $\sigma_{\max }^{e}$, the accuracy of an ANN classifier was significantly boosted to more than $90 \%$ in the sense of modified error rate. Furthermore, by complete the features with $\sigma_{\max }^{e}$, the accuracy was further increased for around $4 \%$ percent and the final result is given in Figure 5. For a better demonstration of the result, we plot samples that have been mistakenly classified with a deviation greater than the tolerance in Figure 6. One can intuitively see from this figure that there are only few errors and all of them are quantitative instead of qualitative. In other words, the confusion occurs only between two neighboring classes. The pattern of classification errors can be seen clearer from the confusion matrix in Figure 7 where all non-zero elements are inside the diagonally bordered band. Based on the confusion matrix, it is justified to conclude that the classifier demonstrates an excellent performance and the pattern of the data have been exploited.

The generality of the ANN classifier has been confirmed through applying it to the test data obtained in another load direction. According to the confusion matrix given in Figure 8, the degree of over-fitting is insignificant. Because the classifier demonstrates sound performance in handling the present classification problem, we established a new ANN in an analogous manner for solving the five-classes classification problem. As expected the performance of the new classifier is satisfactory (see Figure 9), its overall correct rate is $95.8 \%$, and correct rate of the 


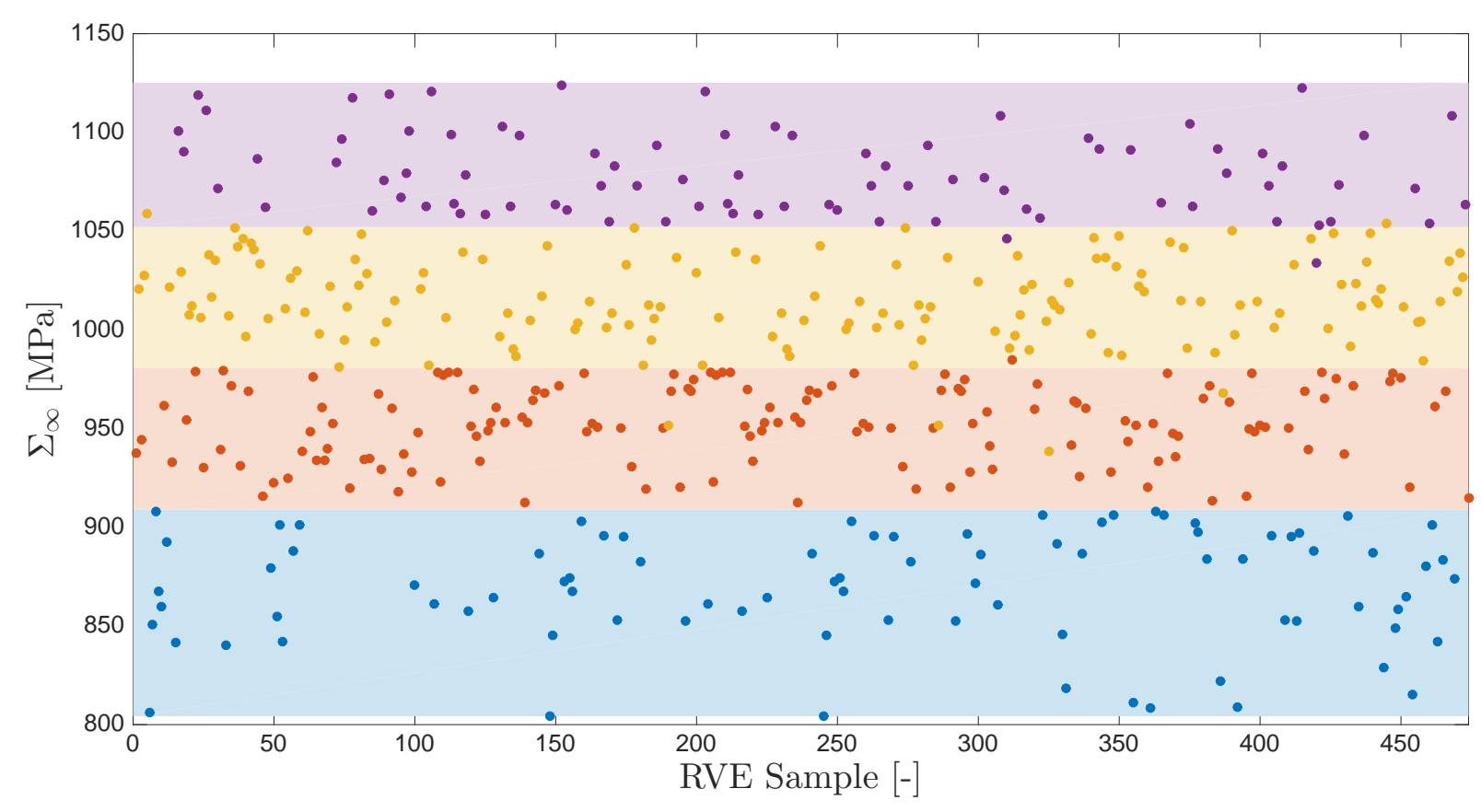

Figure 5: Results of an ANN classification model on $\Sigma_{\infty}$ with features in Category 2

test set is $93.7 \%$. If we set the classification tolerance to $1 / 5 s$, then these two numbers will increase to $97.0 \%$ and $95.9 \%$, respectively.

Although results in Figure 7 were retrieved from an ANN classifier, the accuracy of the classifier built by logistic regression is in the same order where the overall correct rate is about $94.1 \%$, and more than 80 percent of the errors are confusions between two neighboring classes. In addition to that, the over-fitting problem of the logistic regression classifier is insignificant as well. The success of the logistic regression classifier confirms that characteristics of the endurance limit can be well prescribed by linear, interaction, and the squared terms of all considered features.

In summary, the strength class of $\Sigma_{\infty}$ can be accurately predicted from RVE samples by features considered. Among them, the features in Category 2 play a particularly significant role and can fundamentally improve the performance of the classifier.

\section{CONCLUSIONS}

This paper elaborates how to apply shakedown analysis to predict the strength of random PRMMC materials and using statistical models to interpret the results. The study reveals that the endurance limit of the PRMMC material, WC- $20 \mathrm{Wt} \%$ Co, is strongly dependent on the evenness of the effective stress in the binder, and an increased highly stressed volume fraction will lead to a greater endurance limit. The study confirms the benefit of incorporating direct method and statistical learning for studying the strength of random heterogeneous materials. Lastly, one should notice that the failure mechanism considered in the present study is restricted to the plastic failure, more complex failure scenarios, such as plastic damage of the binder phase of the brittle fracture of the reinforcement particles, are not considered and should be investigated in the future. 


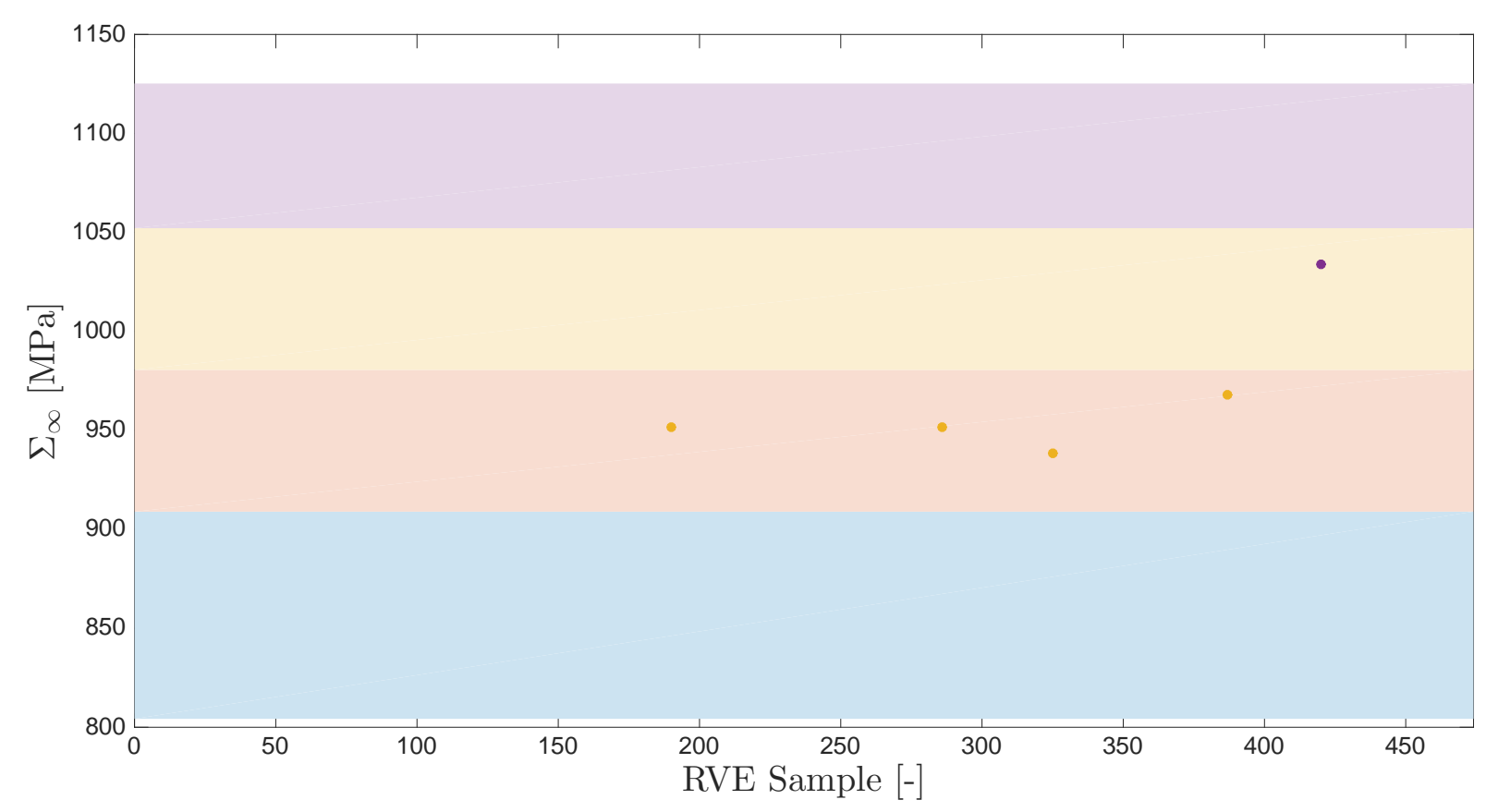

Figure 6: Errors of an ANN classification model on $\Sigma_{\infty}$ with features in Category 2

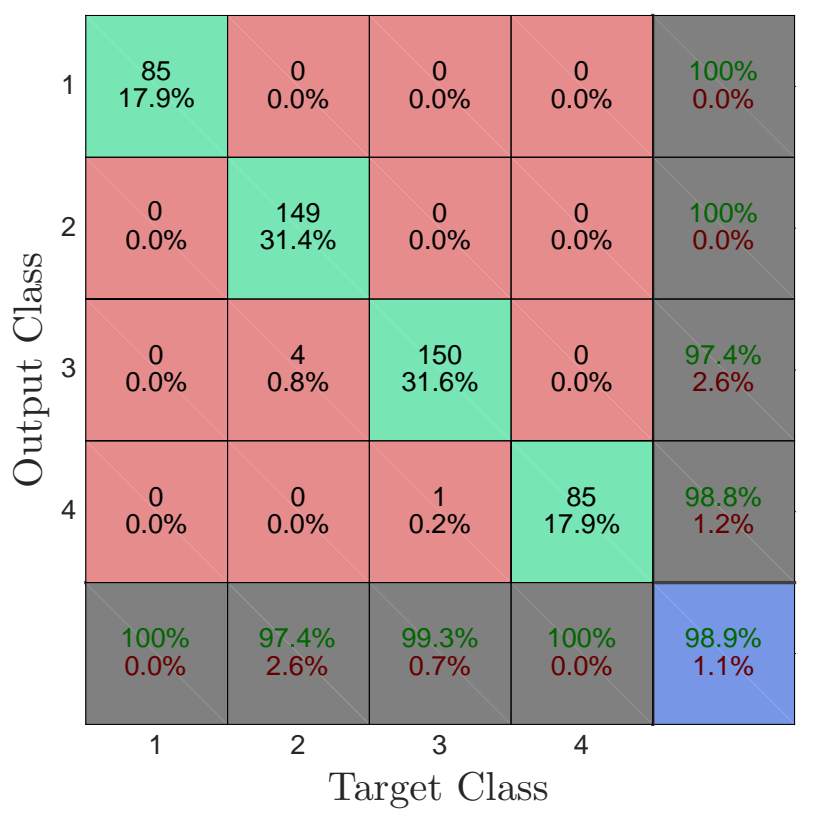

Figure 7: Confusion matrix of an ANN classier on $\Sigma_{\infty}$ with features in Category 2 


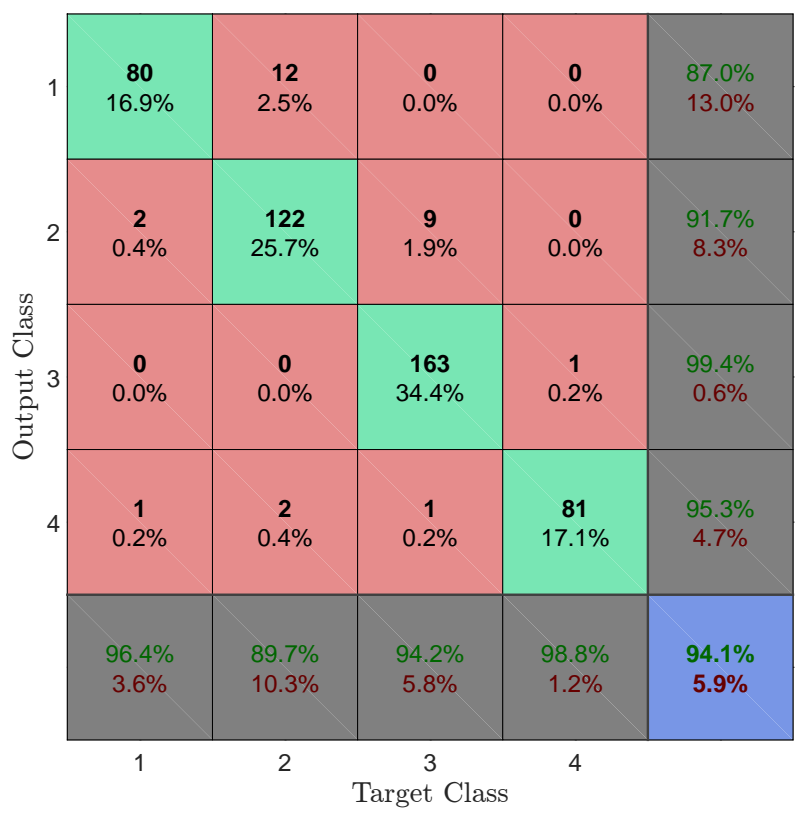

Figure 8: Confusion matrix of an ANN classier on $\Sigma_{\infty}$ applied to test set

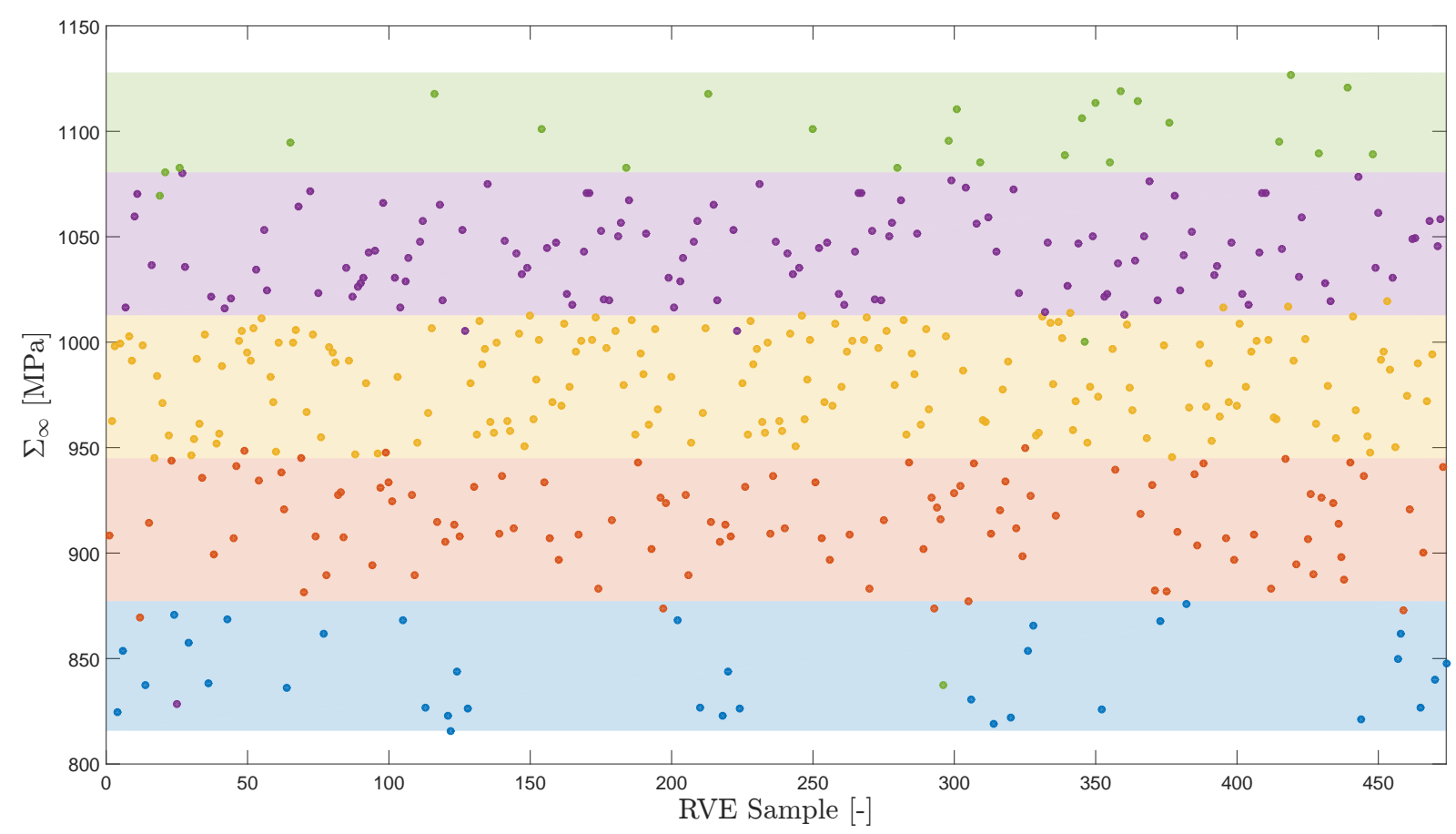

Figure 9: Results of an ANN classification model on $\Sigma_{\infty}$ with features in Category 2 (5 classes)

\section{REFERENCES}

[1] D. Weichert, A. Hachemi, and F. Schwabe. Application of shakedown analysis to the plastic design of composites. Archive of Applied Mechanics, 69(9-10):623-633, 1999.

[2] H.F. Chen and A.R.S. Ponter. Shakedown and limit analyses for 3-D structures using the linear matching method. International Journal of Pressure Vessels and Piping, 78(6):443451, 2001. 
[3] G. Chen, U. A. Ozden, A. Bezold, C. Broeckmann, and D. Weichert. On the statistical determination of yield strength, ultimate strength, and endurance limit of a particle reinforced metal matrix composite (PRMMC). In D. Weichert P. Fuschi, A. A. Pisano, editor, Direct Methods for Limit and Shakedown Analysis of Structures: Advanced Computational Algorithms and Material Modelling, pages 105-122. Springer, 2015.

[4] S.M. Sapuan and I.M. Mujtaba. Composite materials technology: neural network applications. CRC Press, 2009.

[5] Z. Zhang and K. Friedrich. Artificial neural networks applied to polymer composites: a review. Composites Science and Technology, 63(14):2029 - 2044, 2003. Polymer Composites: Design, Materials, Manufacturing, Dedicated to Professor M. Neitzel.

[6] H.E. Kadi. Modeling the mechanical behavior of fiber-reinforced polymeric composite materials using artificial neural networksła review. Composite Structures, 73(1):1 - 23, 2006.

[7] A.O. Addin, S.M. Sapuan, E. Mahdi, and M. Othman. Prediction and detection of failures in laminated composite materials using neural networks-a review. Polymers and Polymer Composites, 14(4):433-442, 2006.

[8] M. Shabani and A. Mazahery. Application of finite element model and artificial neural network in characterization of $\mathrm{Al}$ matrix nanocomposites using various training algorithms. Metallurgical and Materials Transactions A, 43(6):2158-2165, 2012.

[9] M.A. Nik, K. Fayazbakhsh, D. Pasini, and L. Lessard. A comparative study of metamodeling methods for the design optimization of variable stiffness composites. Composite Structures, 107:494 - 501, 2014.

[10] H. Magoariec, S. Bourgeois, and O. Débordes. Elastic plastic shakedown of 3D periodic heterogeneous media: a direct numerical approach. International Journal of Plasticity, 20(8):1655-1675, 2004.

[11] T. Hastie, R. Tibshirani, and J. Friedman. The elements of statistical learning. Springer, 2001.

[12] MATLAB. version 8.4.0 (R2014b). The MathWorks Inc., Natick, Massachusetts, 2014.

[13] ABAQUS. ABAQUS/CAE user's manual : version 6.13. Simulia, Dassault Systémes, 2013.

[14] Gurobi Optimization Inc. Gurobi optimizer reference manual(2014), 2014. 